\title{
Apelin Alleviated Neuroinflammation And Promoted Endogenous Neural Stem Cell Proliferation and Differentiation After Spinal Cord Injury in Rats
}

Qing Liu

Binzhou medical university

\section{Xiao Wang}

Binzhou Medical University

Chengxu Gu

Binzhou Medical University

Qixuan Guo

Binzhou Medical University

Xikai Li

Binzhou Medical University

Chunlei Zhang

Binzhou Medical University

Naili Zhang

Binzhou Medical University

Luping Zhang

Binzhou Medical University

Fei Huang ( $\square$ hfei22518@163.com )

Binzhou Medical University

\section{Research}

Keywords: Apelin, SCl, Neuroinflammation, Endogenous neural stem cells, Astrocytes

Posted Date: November 10th, 2021

DOI: https://doi.org/10.21203/rs.3.rs-1038023/v1

License: (c) (i) This work is licensed under a Creative Commons Attribution 4.0 International License.

Read Full License 


\section{Abstract}

Background: Spinal cord injury (SCl) causes devastating neurological damage, including secondary injuries dominated by neuroinflammation. The role of apelin, an endogenous ligand that binds the $G$ protein-coupled receptor angiotensin-like receptor 1 , in $\mathrm{SCl}$ remains unclear. Thus, our aim was to investigate the effects of apelin in inflammatory responses and activation of endogenous neural stem cells (NSCs) after SCl.

Methods: Apelin expression was detected in normal and injured rats, and roles of apelin in primary NSCs were examined. In addition, we used induced pluripotent stem cells (iPSCs) as a carrier to prolong the effective duration of apelin and evaluate its effects in a rat model of SCl.

Results: Co-immunofluorescence staining suggested that apelin was expressed in both astrocytes and neurons. Following $\mathrm{SCl}$, apelin expression decreased from 1-14 $\mathrm{d}$ and was re-upregulated at $28 \mathrm{~d}$. Apelin promoted NSC proliferation and differentiation into neurons and oligodendrocytes. In vivo, lentiviraltransfected iPSCs were used as a carrier to prolong the effective duration of apelin. Transplantation of transfected iPSCs in situ immediately after SCl reduced activation of microglia and A1 astrocytes, facilitated recovery of motor function, and promoted the proliferation and differentiation of endogenous NSCs in rats.

Conclusion: Apelin alleviated neuroinflammation and promoted the proliferation and differentiation of endogenous NSCs after SCl, suggesting that it might be a promising target for treatment of SCl.

\section{Introduction}

Spinal cord injury (SCl) is a devastating condition affecting millions of people worldwide annually. ${ }^{[1]}$ The prognosis of $\mathrm{SCl}$ is closely related to secondary injury, ${ }^{[2]}$ a process dominated by neuroinflammation. Therefore, inhibiting inflammasome activation, reducing proinflammatory cytokine production, and improving the local spinal cord microenvironment are promising therapeutic strategies for $\mathrm{SCl} .{ }^{[3,4]}$

After $\mathrm{SCl}$, reactive astrocytes form a glial scar that obstructs neuronal axonal regeneration and communication. ${ }^{\left[{ }^{[5]}\right.}$ Astrocytes can reportedly be divided into two types: $A 1$ and $A 2 .{ }^{[6,7]} A 1$ astrocytes release inflammatory cytokines that can inhibit proliferation and differentiation of oligodendrocyte precursor cells, and kill nearby neurons. Thus, it is concluded that inhibiting A1-type reactive astrocytes is an important strategy to suppress inflammation and promote repair of nerve injury. ${ }^{[8]}$

Apelin, an endogenous ligand that binds the G protein-coupled receptor angiotensin-like-receptor 1 (APJ), [9] exhibits a therapeutic effect on central nervous system (CNS) disorders such as stroke, Alzheimer's disease, and Moyamoya disease. ${ }^{[10-12]} \mathrm{A}$ recent study reported that apelin-13 can promote recovery of rat spinal cord ischemia/reperfusion injury by reducing autophagy. ${ }^{[13]}$ However, preliminary studies evaluating the effect of apelin on recovery from $\mathrm{SCl}$ showed that although it was potentially effective, the 
short half-life of apelin peptides greatly limits it therapeutic utility for clinic applications. ${ }^{[14,15]}$ Therefore, it is important to prolong the duration of action of apelin.

Our previous study found that neural stem cells (NSCs) enhance nerve regeneration after sciatic nerve injury in rats. ${ }^{[16]}$ However, the toxic microenvironment of the injury is unfavorable for survival of transplanted cells. ${ }^{[17]}$ Previous studies reported that endogenous NSCs were activated after SCl in the central canal of the spinal cord, whereby they took part in the regeneration of neural function. ${ }^{[18,19]}$ Therefore, promoting the proliferation and differentiation of NSCs can be an effective strategy for treatment of nerve injuries. ${ }^{[20]}$

This study aimed to examine the potential role of apelin in treatment of SCl. First, we evaluated changes of apelin expression after SCI to determine whether apelin was involved in injury or repair processes. Furthermore, to detect the effect of apelin on NSCs, we assessed in vitro proliferation and differentiation of NSCs after administration of apelin or its inhibitor ML221. Finally, to address the short half-life of apelin that greatly limits its therapeutic utility, ${ }^{[21]}$ we infected induced pluripotent stem cells (iPSCs) with lentivirus bearing an apelin expression vector, and then transplanted these cells into the injury in situ to prolong the effective duration of apelin.

Our findings demonstrate that apelin expression was decreased after $\mathrm{SCl}$ from 1-14 days post injury (dpi), and subsequently upregulated at $28 \mathrm{dpi}$. Therefore, we hypothesized that apelin may play a role in cellular and molecular inflammatory cascades of the spinal cord. Our results suggest that apelin contributes to NSC proliferation and differentiation. Furthermore, transplantation of iPSCs overexpressing apelin alleviated inflammation associated with secondary damage; enhanced the recovery of motor functions; reduced $\mathrm{A} 1$ astrocyte activation at $14 \mathrm{dpi}$; and promoted the activation, proliferation, and differentiation of endogenous NSCs in vivo post injury.

In conclusion, our results demonstrate that apelin may be a novel molecular target for SCl recovery.

\section{Materials And Methods}

Animals

A total of 127 specific pathogen-free adult female Sprague-Dawley rats weighing 220-250 g (Jinan PengYue Laboratory Animal Breeding, Jinan, China) were used in experiments. All rats were housed in a separate environment under a 12-h light-dark cycle at $24 \pm 2^{\circ} \mathrm{C}$, with $50 \%$ relative humidity. Food and water were available ad libitum. Experimental protocols for animals in this study were approved by the Animal Care and Use Committee of Binzhou Medical University. All animals were acclimatized to the new environment for at least $7 \mathrm{~d}$ before experiments.

Animal experiments were divided into two parts. Sprague-Dawley rats for Part 1 were randomized into three groups: control $(n=6)$, sham control $(n=6)$, and $\mathrm{SCl}(n=30)$. Rats in the SCI group were sacrificed at $1,3,7,14$, and $28 \mathrm{dpi}$. Rats in Part 2 were randomized into five groups: (1) Sham: rats subjected to 
laminectomy only; (2) SCl: after laminectomy, a transection was made between T9 and T10 with a sharp blade, and $4 \mu \mathrm{L}$ of iPSC culture medium were locally injected immediately after SCl; (3) H-Apln: $1 \times 10^{5} \mathrm{H}-$ Apln iPSCs (Apln-overexpressing iPSCs) were locally injected immediately after SCl; (4) Green fluorescent protein (GFP): $1 \times 10^{5}$ GFP+ iPSCs (iPSCs infected with a GFP vector only) were locally injected immediately after SCl; and (5) ML221: ML221 (apelin inhibitor, $30 \mu \mathrm{g} / \mathrm{rat}$ ) was intraperitoneally administered 30 min after $\mathrm{SCl}$.

$\mathrm{SCl}$ surgery, cell transplantation, and inhibitor injection

The SCI transection model was established as previously reported. ${ }^{[22]}$ Briefly, rats were anesthetized with $4 \%$ chloral hydrate (100 mg/kg body weight; Tianjin DaMao Chemical Reagent Factory, Tianjin, China). The spinal cords of sham group rats were exposed at T8-T10 by laminectomy of these vertebrae. For the $\mathrm{SCl}$ transection model, the spinal cord was transected at T9 using a No. 11 blade, and the bleeding was controlled using gauze. SCl group rats were immediately locally injected with $4 \mu \mathrm{L}$ of iPSC culture medium using a microsyringe, while H-Apln group and GFP group rats were given the same volume of culture medium containing $5 \times 10^{5}$ iPSCs (Apln-overexpressing or GFP-infected only, respectively). After surgery, urination was aided twice a day until recovery of the micturition reflex.

Tissue processing

At designated time points post-injury, rats were anesthetized with $4 \%$ chloral hydrate and intracardially perfused with at least $200 \mathrm{~mL}$ of $0.9 \%$ physiological saline, followed by $400 \mathrm{~mL}$ of paraformaldehyde (PFA). Subsequently, 1-cm segments were obtained from the injured lesion.

Paraffin section histopathological staining

At designated time points after injury, the tissue around the injury site at T8-T10 (about $1 \mathrm{~cm})$ was obtained and fixed in PFA for at least $48 \mathrm{~h}$. Subsequently, the tissue was dehydrated in xylene followed by a gradient series of alcohol, embedded in paraffin, cut into $4-\mu \mathrm{m}$ serial sections, heated at $60^{\circ} \mathrm{C}$ for at least $2 \mathrm{~h}$, and then stored at room temperature.

Hematoxylin and eosin (HE) staining

Paraffin sections were placed in xylene followed by a gradient series of alcohol, and then stained with hematoxylin for $5 \mathrm{~min}$ at room temperature. After rinsing sections in running water, they were differentiated in $1 \%$ hydrochloric acid and double-stained with eosin for 3 min. Finally, slides were dehydrated with a gradient of ethanol, permeabilized with xylene, and sealed.

Luxol Fast Blue (LFB) staining

Paraffin sections were placed in xylene, followed by $100 \%$ and $95 \%$ ethanol solutions. Next, sections were stained with LFB solution and heated at $60^{\circ} \mathrm{C}$ overnight. The next day, sections were washed in $95 \%$ ethanol and then placed in $\mathbf{0 . 0 5 \%}$ lithium carbonate aqueous solution for $10 \mathrm{~s}$, followed by $75 \%$ alcohol 
for $30 \mathrm{~s}$; the last two steps were repeated until the white and grey matter were clearly observable. Finally, slides were sealed after dehydration in ethanol and xylene.

Nissl staining

Paraffin sections were dewaxed and rehydrated before staining with cresyl violet. Subsequently, sections were differentiated in $75 \%$ ethanol solution and distilled water until Nissl bodies were clearly observable under a microscope. Numbers of Nissl bodies in the anterior horn were used to detect neuronal damage.

Frozen section preparation

Spinal cord tissues around the injured lesion were placed into PFA overnight at $4^{\circ} \mathrm{C}$, dehydrated with sucrose solution ( $15 \%$ for $1 \mathrm{~d}$ and $30 \%$ for $2 \mathrm{~d}$ ), embedded in optimum cutting temperature compound, and cut into $12-\mu \mathrm{m}$ frozen sections with a microtome.

Immunofluorescence

Frozen sections were equilibrated to room temperature (RT), washed three times (15-min each) with phosphate-buffered saline ( $\mathrm{pH} 7.2-7.4)$, blocked for $1 \mathrm{~h}$ with normal goat serum at RT, and incubated with primary antibody at $4^{\circ} \mathrm{C}$ overnight. Following overnight incubation, sections were washed three times and incubated with secondary antibody for $2 \mathrm{~h}$ at RT. Finally, sections were washed another three times, nuclear stained with 4,6-diamidino-2-phenylindole (DAPI) for 8 min at RT, and sealed with an antifluorescence quencher. Images were obtained under a fluorescent microscope.

The following primary antibodies were used for immunofluorescence at the indicated dilutions: antiapelin (1:400; Affinity Biosciences, Zhenjiang, China), anti-glial fibrillary acidic protein (GFAP;ab7260, 1:500; Abcam, Cambridge, UK), anti-GFAP (ab4674,1:1000, Abcam), anti-lba1 (1:200, Abcam), anti-Olig2 (1:200; R\&D Systems, Minneapolis, MN, USA), anti-NeuN (1:400; Proteintech, Rosemont, IL, USA), antinestin (1:300, Proteintech), anti-C3 (1:200, Proteintech), anti-CD68 (1:200, Abbkine, Wuhan, China), antiBrdU (1:300, Abbkine), anti-NeuN (1:50; Cell Signaling Technology, Danvers, MA, USA).

The following fluorescent secondary antibodies were used at a suitable concentration: Alexa Fluor 488 goat-anti-rabbit (Invitrogen, Carlsbad, CA, USA), Alexa Fluor 594 goat-anti-mouse (Abbkine), Alexa Fluor 555 goat-anti-chicken (Bioss, Beijing, China), Alexa Fluor 594 rabbit-anti-goat (Abbkine), and Alexa Fluor 350 goat-anti-rabbit (Abbkine).

Western blotting

Total protein was extracted from spinal cord tissue using radioimmunoprecipitation assay buffer containing $1 \%$ phenylmethanesulfonylfluoride. Protein concentrations were measured using a BCA assay kit (Beyotime, Shanghai, China). Proteins were resolved by sodium dodecyl sulfate polyacrylamide gel electrophoresis (using gels of different concentrations) and subsequently transferred onto polyvinylidene difluoride membranes. Next, membranes were incubated with the following primary antibodies at $4^{\circ} \mathrm{C}$ 
overnight: anti-apelin (1:1000; Affinity Biosciences, Zhenjiang, China), anti-nestin (1:2000, Proteintech), anti-GFAP (1:4000, Abcam), anti-C3 (1:1000, Proteintech), and anti-lba1 (1:1000, Abcam), as well as antiGAPDH (1:10000, Proteintech) as an internal control. The following day, membranes were washed three times with Tris-buffered saline containing Tween (TBST, 15 min each), incubated with secondary antibodies at room temperature for $2 \mathrm{~h}$, and then washed three times with TBST. Finally, protein bands were visualized using a Bio-Rad Image Lab system (Hercules, CA, USA), and densitometry analysis was performed with ImageJ software (http://imagej.nih.gov).

Quantitative Reverse Transcription PCR (qRT-PCR)

Total RNA was extracted from spinal cord tissue with TRIzol (Takara, Kusatsu, Japan) according to the manufacturer's protocol. To assess the purity and concentration of total RNA templates, 260/280 and 260/230 absorbance ratios were determined using an ultraviolet-visible light spectrophotometer (NanoDrop 2000; Thermo Fisher Scientific, Waltham, MA, USA). A TranscriptorFirst Strand cDNA Synthesis Kit (Roche, Basel, Switzerland) was used to synthesize cDNA from total RNA (1000 $\mathrm{ng} /$ sample). qRT-PCR was carried out using Premix Ex Taq ${ }^{\text {TM }}$ (Probe qPCR) (Roche) with GAPDH as an internal reference gene. All primers (Table 1 for detailed primer information) were purchased from Accurate Biotechnology (Hunan, China). Data were analyzed by the $2^{-\triangle \Delta C T}$ method.

Table 1

Gene primers tested in comparative RT-PCR experiments

\begin{tabular}{|c|c|c|}
\hline Gene & Forward primer $\left(5^{\prime}-3^{\prime}\right)$ & Reverse primer $\left(5^{\prime}-3^{\prime}\right)$ \\
\hline Apln & CGATGGGAATGGGCTGGAAGA & CAGAAAGGCATGGGTCCCTTATG \\
\hline Beta- $囚$-tubulin & CAGATGCTGGCCATTCAGAGTAAG & TGTTGCCGATGAAGGTGGAC \\
\hline Olig2 & ACCGAAGTAGTGAGAGCACTTGGAG & ATGGATGTACCCGCGTGTTG \\
\hline Iba1 & GAGGCCTTCAAGACGAAGTACA & GGGAACCCCAAGTTTCTCCAG \\
\hline GFAP & GCCACCTCAAGAGGAACATCG & СTTGTGCTCCTGCTTCGACTC \\
\hline IL-1 beta & СССТGAACTCAACTGTGAAATAGCA & CCCAAGTCAAGGGCTTGGAA \\
\hline $\mathrm{IL}-4$ & TGCACCGAGATGTTTGTACCAGA & TTGCGAAGCACCCTGGAAG \\
\hline IL-10 & CAGACCCACATGCTCCGAGA & CAAGGCTTGGCAACCCAAGTA \\
\hline TNF-alpha & TCAGTTCCATGGCCCAGAC & GTTGTCTTTGAGATCCATGCCATT \\
\hline GAPDH & GCACCGTCAAGGCTGAGAAC & TGGTGAAGACGCCAGTGGA \\
\hline
\end{tabular}

Enzyme-linked immunosorbent assay (ELISA)

Spinal cord tissues were homogenized in phosphate-buffered saline, followed by centrifugation at $5000 \times \mathrm{g}$ for $10 \mathrm{~min}$ to extract protein samples. Protein concentrations in each sample were measured with a BCA assay kit (Beyotime) and adjusted to the same concentration. Apelin expression was measured in 
serum, while interleukin 1 beta (IL-1 3 ), IL-10, and tumor necrosis factor alpha (TNF- $\alpha$ ) were measured in tissue with ELISA kits (Could-Clone Crop, Wuhan, China) according to the manufacturer's protocols. After detecting the absorbance at a 450-nm wavelength, the concentration of each factor was calculated based on a standard curve.

iPSC culture and transfection

Human iPSCs (Saibaikang Biotechnology, Shanghai, China) were cultured in mTeSR1 medium. iPSCs were transfected with lentivirus stably expressing Apln or GFP only (Jiman Gene, Shanghai, China). Stably transfected cells were selected by puromycin (MedChemExpress, Monmouth Junction, NJ, USA) before use.

NSC cultures from newborn rats

Primary spinal cord NSC cultures were prepared from newborn Sprague-Dawley rats ( $<24 \mathrm{~h})$ as previously described. ${ }^{[23]}$ Briefly, newborn rats were anesthetized with isoflurane and decapitated. Spinal cord tissues were manually freed of meninges under a stereoscopic microscope, cut into small pieces (approximately $1-\mathrm{mm}^{3}$ ), and then separated into cells by repeated pipetting using a $5-\mathrm{mL}$ pipette until no macroscopic tissue was observed. Next, the suspension of NSCs was filtered through a $0.22-\mu \mathrm{m}$ filter and centrifuged at $800 \times \mathrm{g}$ for 6 min at RT. Finally, $5 \times 10^{5}$ cells in suspension were transferred into a T25 culture flask and cultured at $37^{\circ} \mathrm{C}$ with $5 \% \mathrm{CO}_{2}$ (Thermo Fisher Scientific) for $7 \mathrm{~d}$. NSC purity was confirmed by staining for nestin. After $48 \mathrm{~h}$ in differentiation conditions, GFAP immunocytochemistry was performed to evaluate the multi-directional differentiation ability of NSCs. ${ }^{[24]}$ Cells in the third to fifth passage were used for experiments.

NSC differentiation

To evaluate the effect of apelin and its inhibitor ML221 on NSC differentiation, NSCs were cultured on poly-L-lysine (PL)-coated dishes with different dosages of apelin or its inhibitor ML221. Subsequently, immunocytochemistry using antibodies against GFAP (astrocyte marker), Iba1 (microglia marker), Olig2 (oligodendrocyte marker), and NeuN (neuron marker) was performed to assess NSC differentiation. In addition, mRNA levels of these markers was examined using specific primers and qRT-PCR, as described above.

Cell counting kit-8 (CCK8) assay

CCK8 assay was performed with a CCK8 kit (Beyotime) according to the manufacturer's protocol. Briefly, $2 \times 10^{4} \mathrm{NSC}$ in $100 \mu \mathrm{L}$ of induction medium were seeded in PL-coated 96-well plates, to which different dosages of apelin or ML221 were added. After culture for $24 \mathrm{~h}, 48 \mathrm{~h}$, or $14 \mathrm{~d}$, CCK8 detection was performed by adding $10 \mu \mathrm{L}$ of CCK8 reagent to each well for $1 \mathrm{~h}$ before analysis. Cell proliferation rates were calculated as the absorbance of treated cells compared with an untreated control group. 
Basso-Beattie-Bresnahan (BBB) scores

BBB scores ${ }^{[25]}$ were used to evaluate rat hindlimb motor function at $1,3,7$, and 14 dpi by two researchers who did not otherwise participate in experiments.

Statistical analyses

Quantification was performed by researchers blinded to the present study, and experiments were independently conducted three times with at least six replicates for each group. The resulting data are expressed as the mean \pm standard deviation (SD), as calculated by Prism 8.0.1 (GraphPad Software, San Diego, CA, USA). Student's t-test was used to compare two groups. For multiple comparisons, data were analyzed by one-way ANOVA followed by Tukey's post hoc test. Assessment of BBB scores was analyzed by two-way RM ANOVA. P $<0.05$ was considered statistically significant.

\section{Results}

Apelin expression was decreased after SCI

To evaluate apelin expression after $\mathrm{SCl}$, spinal cord tissues and serum from rats in normal, sham, and $\mathrm{SCl}$ groups were collected at 1, 3, 7, 14 and 28d. Western blotting and RT-PCR results indicated that both protein and mRNA levels of apelin decreased from 1-14 dpi (lowest level at $14 \mathrm{dpi}$ ) compared with normal and sham groups, then increased at $28 \mathrm{dpi}\left({ }^{*} \mathrm{P}<0.05, * \star P<0.01\right.$, Figure $\left.1 \mathrm{~A}, \mathrm{~B}\right)$. Results of ELISA to quantify apelin contents in peripheral blood showed the same results $(* P<0.05, \star * P<0.01$, Figure $1 \mathrm{C})$. Collectively, these results suggest that apelin might be a candidate molecule related to $\mathrm{SCl}$.

Cellular distribution of apelin after SCl

To investigate which cell types express apelin in spinal cord tissue, double immunofluorescence staining was performed after SCl; colocalization was indicated as a yellow fluorescent signal. As shown in Figure 2A, apelin was distributed in cells positive for NeuN or GFAP, but not CD68, suggesting that apelin was located in both neurons and astrocytes, but not microglia.

Next, to evaluate apelin expression levels in different cell types after SCl, we obtained the spinal cord tissue at $1,3,7,14$ and $28 d$.

Immunofluorescence results revealed apelin expression in NeuN-positive cells of rats in normal and sham groups, but not the SCI group at $14 \mathrm{dpi}$ (Figure 2B). Statistical analysis showed that apelin expression in neurons and astrocytes gradually decreased after $\mathrm{SCl}$, reached its lowest point at $14 \mathrm{dpi}$ and then increased after $28 \mathrm{dpi}\left({ }^{\star} \mathrm{p}<0.05,{ }^{*} \mathrm{p}<0.01\right.$, Figure $\left.2 \mathrm{~B}-\mathrm{E}\right)$.

Characterizations of NSCs 
To characterize NSCs extracted from newborn rats, spherical neurospheres cultured in suspension were evaluated with fine light refraction under a light microscope (Figure 3A). After seeding in PL-coated plates at $37^{\circ} \mathrm{C}$ for $4 \mathrm{~h}$, cells exhibited radial growth around the neurospheres. NSCs marker were employed to identify NSCs (Figure 3B). Next, we evaluated the differential potency of NSCs by fluorescent staining of cells with GFAP (an astrocyte marker). To evaluate differentiation potential, differentiated astrocytes were identified (Figure 3C). NSCs from third to fifth generations were collected for subsequent experiments.

Apelin treatment promoted NSC proliferation

To investigate whether apelin and its inhibitor ML221 could affect NSC proliferation, we conducted CCK8 assays. Third-passage NSCs were seeded into 96-well plates, and proliferation was evaluated $24 \mathrm{~h}, 48 \mathrm{~h}$, and $14 \mathrm{~d}$ after the addition of each drug. As shown in Figure 3D, after $24 \mathrm{~h}$, no significant proliferation was detected. However, CCK8 assay results after $48 \mathrm{~h}\left({ }^{\star} \mathrm{p}<0.05,{ }^{*} \mathrm{p}<0.01\right.$, Figure $3 \mathrm{E}$ ) indicated that cellular proliferation was significantly promoted by $2 \mu \mathrm{mol}$ and $4 \mu \mathrm{mol}$ apelin, whereas ML221 administration did not elicit a statistically significant effect. After $14 \mathrm{~d}$ (Figure 3F), $4 \mu \mathrm{mol}$ apelin promoted NSC proliferation while $2 \mu \mathrm{mol}$ ML221 inhibited proliferation. Thus, $4 \mu \mathrm{mol}$ apelin and $2 \mu \mathrm{mol}$ ML221 were selected as optimal dosages for subsequent experiments.

Apelin and its inhibitor ML221 altered NSC differentiation

To evaluate NSC differentiation after administration of apelin or its inhibitor ML221, various dosages of apelin and ML221 were added to the differentiation medium. As shown in Figure 4A-D, after 14-d administration, apelin promoted differentiation of NSCs into astrocytes (GFAP, green; Figure 4A), microglia (Iba1, red; Figure 4B), oligodendrocytes (Olig2, red; Figure 4C), and neurons (NeuN, green, arrowheads; Figure 4E). To quantitatively analyze differentiation, qRT-PCR was conducted. The results indicated that apelin promoted NSC differentiation into oligodendrocytes ( $* P<0.05$, Figure $4 G$ ) and neurons (beta-three-tubulin; ${ }^{* * P}<0.01$, Figure $4 \mathrm{H}$ ), reduced NSC differentiation into microglia (Iba1; ** $\mathrm{P}<$ 0.01 , Figure 4F), and had no significant effect on astrocytes (GFAP, Figure 4E). Conversely, ML221 administration promoted differentiation of NSCs into astrocytes (GFAP; $* \star \star P<<0.001$, Figure 4E), but elicited no statistically significant differences for the other three cell types.

iPSC transplantation promoted morphological and functional recovery after SCI

To determine the neuroprotective effect of transplanting lentivirus-infected iPSCs after SCl, rats were sacrificed at $14 \mathrm{~d}$ post injury. Next, animals were sacrificed and their spinal cord tissues were collected. Morphometric analysis of HE, LFB, and Nissl staining of paraffin-embedded spinal cord tissue was performed. As shown in Figure 5A, syringomyelia formed in longitudinal sections post $\mathrm{SCl}$, but transplantation of iPSCs improved spinal cord tissue recovery after SCl in GFP and H-Apln groups.

Results of LFB staining to evaluate myelination (Figure 5B) showed that demyelination and degeneration occurred after SCl. Moreover, the $\mathrm{H}$-Apln group exhibited significantly increased formation of myelin 
sheaths during SCl. However, administration of ML221 yielded more LFB-unstained areas compared with the $\mathrm{SCl}$ group, suggesting more severe demyelination.

Nissl bodies were used as an indicator of neuronal survival. Nissl staining results indicated significant neuronal loss after $\mathrm{SCl}$ in the spinal cord anterior horn ( ${ }^{* \star} \mathrm{P}<0.001$ vs. Sham group; Figure $5 \mathrm{C}, \mathrm{D}$ ). After transplantation of iPSCs overexpressing apelin, the number of neurons was significantly increased ( $\star \star P<$ $0.01,{ }^{*} * \mathrm{P}<0.001$ vs. Sham group; Figure $5 \mathrm{D}$ ), but no statistical difference was observed between $\mathrm{H}$-Apln and Sham groups. After intervention with the apelin inhibitor ML221, the number of neurons was significantly decreased.

We followed up with behavioral testing at 1, 3,7 and $14 \mathrm{~d}$. BBB scores (Figure 5E) suggested that iPSC administration promoted the functional recovery of rat hindlimbs after 3 dpi compared with the SCI group. Indeed, BBB scores of rats transplanted with iPSCs overexpressing apelin were higher than SCI rats, while rats administered ML221 exhibited no functional changes.

In addition, $\mathrm{SCl}$ led to morphological degeneration of the spinal cord, which might form the basis of motor degeneration. These changes were reversed by iPSC transplantation, especially in the H-Apln group, indicating that apelin might be an attractive target for SCl.

Transplantation of iPSCs prevented microglial activation and alleviated neuroinflammation

It was previously reported that microglia activation is the major source of neuroinflammation, ${ }^{[26]}$ therefore, we assessed microglial activation and morphological changes by immunofluorescence

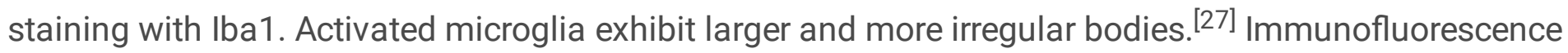
results indicated an increased number of microglia in the $\mathrm{SCl}$ group compared with the sham group, which exhibited the morphology of activated microglia (Figure 6A). After iPSC transplantation, numbers of microglia were decreased in GFP and H-Apln groups, and these cells had smaller bodies and longer processes. After $14 \mathrm{dpi}$, microglia in the ML221 group were similar to those of the $\mathrm{SCl}$ group. Western blotting and qRT-PCR yielded similar results (Figure 6C, D).

Next, we evaluated levels of pro- and anti-inflammatory factors by RT-PCR (Figure 6B). Because they can induce microglia to adopt M1 or M2 phenotypes, IL-4 and IL-10 were selected as representative antiinflammatory factors, and IL-1 $\beta$ and TNF- $a$ were selected as pro-inflammation cytokines, respectively. ELISA results showed that transplantation of apelin overexpressed iPSCs reversed the release of proinflammatory mediators and promoted release of anti-inflammatory factors $\left({ }^{\star} P<0.05,{ }^{*} P<0.01\right.$, ${ }^{\star \star \star} P$ $<0.001$ vs. Sham group; ${ }^{\# \#}$ < 0.01, ${ }^{\# \# \# ~} \mathrm{P}<0.001$, GFP vs. H-Apln; Figure 6B).

Transplanted cells suppressed activation of A1 astrocytes

As described in the introduction, astrocytes can be divided $\mathrm{A} 1$ and $\mathrm{A} 2$ astrocytes. ${ }^{[28]} \mathrm{A} 1$ reactive astrocytes display neurotoxic properties and can induce neuronal degeneration. ${ }^{[29]}$ In contrast, A2 astrocytes can elicit neuroprotective effects. In our study, we focused on A1 astrocytes to evaluate 
neuroinflammation. As previously reported, A1 astrocytes were activated by pro-inflammatory cytokines secreted by activated microglia, such as IL-1, TNF-a, and C1q. ${ }^{[8]}$ Therefore, ELISA assays were used to detect levels of these cytokines in spinal cord tissue. As shown in Figure 7A-C, very few pro-inflammatory cytokines were observed in the Sham group, but these cells were increased dramatically in the SCI group

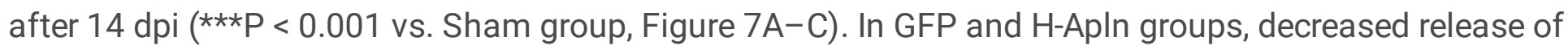
pro-inflammatory factors was observed compared with the SCI group. Expression levels of IL-1 and C1q did not differ between these two groups, but expression of TNF-a was decreased in the H-Apln group compared with the GFP group ( ${ }^{*} \mathrm{P}<0.05$, Figure $7 \mathrm{C}$ ), indicating that transplanted iPSCs overexpressing apelin yielded a better anti-inflammatory effect.

Complement component 3 (C3) is a characteristic marker of astrocytes; ${ }^{[30]}$ accordingly, we identified A1 astrocytes using co-immunofluorescence staining for GFAP and C3. As shown in Figure 7D, GFAP (red)/C3 (blue) double-positive cells were rarely seen in the Sham group, but rapidly increased in number in the $\mathrm{SCl}$ group. iPSC administration significantly decreased the level of $\mathrm{C} 3$, a phenomenon that was particularly evident in the H-Apln group. The results of western blotting to quantify protein expression of C3 and GFAP were consistent with immunofluorescence. In addition, administration of apelin overexpressed iPSCs suppressed activation of A1 astrocytes, resulting in an anti-inflammatory effect.

Transplantation of iPSCs overexpression apelin promoted activation, proliferation, and differentiation of endogenous NSCs

Previous reports suggest that activation and migration of endogenous NSCs following SCl plays a key role in spontaneous self-repair. ${ }^{[31,32]}$ Therefore, we first used BrdU (50 mg/kg) incorporation to evaluate NSC proliferation. Immunofluorescence results revealed that transplantation of iPSCs increased the expression of nestin/BrdU double-positive cells, suggesting that transplantation enhanced NSC activation and proliferation after SCl (Figure 8A). Similar results were obtained with western blotting (Figure 8D).

We next examined numbers of NeuN/BrdU double-positive cells (newborn neurons, Figure 8B) and GFAP/BrdU double-positive cells (newborn astrocytes, Figure $8 \mathrm{C}$ ). Our results indicate that numbers of NeuN/BrdU double-positive cells were increased in the H-Apln group (arrowheads, Figure 8B), however no differences were observed in the other groups. For GFAP/BrdU double-positive cells, numbers were increased significantly after SCl. Transplantation of iPSCs overexpression apelin suppressed differentiation of NSCs toward astrocytes (asterisks, Figure 8C). Among injured groups, the lowest number of astrocytes was observed in the H-Apln group.

Thus, we concluded that administration of iPSCs overexpressing apelin can promote NSC differentiation into new neurons and suppress NSC differentiation towards astrocytes. Notably, this effect was most pronounced in the H-Apln group.

\section{Discussion}


$\mathrm{SCl}$, a severe CNS injury that can result in motor and sensory disorders, ${ }^{[33]}$ can be divided into primary and secondary injury. ${ }^{[34]} \mathrm{SCl}$ remain a challenging neurological disorder for which there is currently no cure. Therefore, it is of great importance to identify novel therapies.

Secondary injury is a main contributor that leads to long-term damage. Inflammatory responses contribute to neural injury phases and aggravate tissue injury. After CNS injury, astrocytes are activated by neuroinflammatory factors such as IL-1, TNF-a, and C1q secreted by microglia ${ }^{[28]}$ in a process termed "astrogliosis" ${ }^{[6,7]}$ As previously reported, $A 1$ astrocytes can secrete neurotoxins that induce neuronal and oligodendrocyte cell death. ${ }^{[28,35,36]}$ Moreover, astrogliosis leads to the formation of glial scars - a major inhibitor of axonal regeneration. Therefore, decreasing A1 astrocyte reactivity and secretion of proinflammation cytokines might be a promising strategy to alleviate functional loss after SCl.

Apelin, an endogenous peptide that binds the apelin receptor APJ, can contribute to traumatic brain injury by suppressing autophagy, ${ }^{[37-39]}$ neuronal apoptosis, and inflammation. In addition, apelin can reduce reactive oxygen species accumulation; ${ }^{[37]}$ lower expression of pro-inflammation cytokines such as IL-1, IL-10, and TNF-a; increase Bax/Bcl2 expression ratios; and reduce numbers of terminal deoxynucleotidyl transferase dUTP nick end labeling-positive cells. Another report suggests that intranasal delivery of apelin-13 could promote angiogenesis and elicit neuroprotective effects after ischemic stroke in mice, ${ }^{[40]}$ indicating that apelin can promote angiogenesis. Similar observations were made in the cardiovascular and reticuloendothelial systems. ${ }^{[41]}$ Apelin has also been implicated in many neurological disorders such as Alzheimer's, Parkinson's, and Moyamoya diseases. ${ }^{[42-46]}$ Taken together, apelin/APJ has unique functions in regulating cell proliferation, apoptosis, pro-inflammatory activity, and revascularization. Therefore, we speculated that apelin has similar functions in SCl.

Although several studies have investigated the effect of apelin on neural system injury, we focused on its role in SCl. A previous study reported that apelin could alleviate spinal cord ischemia/reperfusion injury by suppressing autophagy, ${ }^{[47]}$ while another found that intraperitoneal apelin administration could suppress secretion of pro-inflammatory cytokines and promote behavioral recovery of rats with spinal cord contusion. ${ }^{[48]}$ To verify the effect of apelin, intracerebroventricular administration and intranasal delivery of apelin-13 have also been used as delivery modes. ${ }^{[40,49]}$ However, because the half-life $(\mathrm{t} 1 / 2)$ of apelin was previously noted to be $<5 \mathrm{~min},{ }^{[15]}$ it is necessary to find a new delivery system. Previously, a novel liposomal nanocarrier system was used to deliver and prolong the duration of [Pyr1]-apelin-13 as a therapeutic molecule into the injury site of a mouse model of transverse aortic constriction. ${ }^{[50]}$ In our study, we attempted to use iPSCs as a carrier for drugs with a short half-life.

To clarify the effect of apelin on $\mathrm{SCl}$, we first detected spatiotemporal variations in apelin expression after $\mathrm{SCl}$. Our results show that apelin expression decreased after $\mathrm{SCl}$ compared with normal and sham groups. The lowest expression was detected at $14 \mathrm{dpi}$, and subsequently apelin was re-upregulated at 28 dpi. Next, we found that apelin was located in neurons and astrocytes, suggesting its potential involvement in SCl. 
Stem cell therapy is considered an effective method for treating SCI. ${ }^{[51]}$ However, some reports suggest that stem cells transplanted after $\mathrm{SCl}$ survive only 1-2 weeks. Accordingly, promoting proliferation and differentiation of endogenous NSCs might be a promising approach. ${ }^{[52]}$ Hence, we extracted NSCs from rat spinal cord and treated them with different dosages of apelin or its inhibitor ML221 to evaluate effects on NSC proliferation and differentiation. Our results show that $4 \mu \mathrm{mol}$ apelin-13 promoted NSC differentiation into neurons and oligodendrocytes, and reduced NSC differentiation into astrocytes and microglia. CCK8 assay results also indicated that apelin could promote NSC proliferation. Collectively, the results of these in vitro experiments lay the groundwork for further in vivo studies.

To further detect the efficacy of apelin in vivo, we intraspinally administered apelin immediately after injury. However, because apelin has a short half-life in the body, as low as $5 \mathrm{~min},{ }^{[15]}$ we used iPSCs as a carrier. Lentivirus-infected iPSCs overexpressing apelin were transplanted into the injured spinal cord to observe treatment effects.

Our findings show that our administration alleviated the morphological and functional alterations induced by SCl. Moreover, HE staining indicated that this intervention augments spinal cord tissue repair. Nissl staining showed that transplantation of iPSCs overexpressing apelin increased the number of Nissl bodies, indicating alleviation of neuronal injury. In addition, LFB staining suggested an increase in myelinated axons following iPSC transplantation. In sum, our study indicated that iPSCs overexpressing apelin could alleviate morphological damage and improve motor function in the hindlimbs (BBB scores) of rats.

After $\mathrm{SCl}$, excessive activation astrocytes can be induced. ${ }^{[53]}$ As previously reported, astrocytes play multifaceted roles after SCI. ${ }^{[54]}$ On one hand, the response of astrocytes is important for restricting neuroinflammation and restoring tissue integrity during the acute phase of $\mathrm{SCl}^{[55]}$ On the other hand, excessive activation of astrocytes is related to glial scar formation, which is thought to prevent axonal regeneration. ${ }^{[56]}$ However, recent studies indicate that astrocytes can be divided into two phenotypes: A1 neurotoxic astrocytes and $A 2$ neuroprotective astrocytes. ${ }^{[57]} \mathrm{A} 1$ astrocytes are activated by proinflammation cytokines including IL-1, TNF-a, and C1q, which are secreted by microglia; ${ }^{[58]}$ thus, we used ELISA to detect levels of these cytokines. Our results indicate that these pro-inflammatory factors were significantly decreased in GFP and H-Apln groups; moreover, TNF-a was lower in the GFP group compared with the H-Apln group. Recent studies reported that $\mathrm{C} 3$ is expressed on A1, but not A2, astrocytes; therefore, we determined numbers of A1 astrocytes by C3/GFAP double-positive immunofluorescence. ${ }^{[59]}$ Our results show that numbers of C3/GFAP double-positive cells in the $\mathrm{H}$-Apln group were lower compared with other injured groups; western blotting results further supported these results. In addition, transplantation of iPSCs overexpressing apelin reduced the secretion of pro-inflammation factors and numbers of $\mathrm{A} 1$ reactive astrocytes, thereby alleviating the inflammatory response.

Microglia, the most important effector cells after $\mathrm{SCl}$, can be divided into $\mathrm{M} 1$ and $\mathrm{M} 2$ phenotypes. ${ }^{[60,61]}$ The M1 phenotype is associated with production of multiple pro-inflammatory factors, such as IL-1 $\beta$ and 
TNF-a. In contrast, anti-inflammatory cytokines IL-4 and IL-10 are important factors associated with antiinflammatory M2 macrophages. ${ }^{[62,63]}$ Hence, we evaluated gene expression of IL-4, IL-10, TNF-a, and IL$1 \beta$ by qRT-PCR to examine the polarization state of microglia. Our results demonstrate that transplantation of apelin overexpressed iPSCs downregulated pro-inflammatory cytokines and upregulated anti-inflammation cytokines. Next, we detected Iba1 protein levels by western blotting and immunofluorescence. Numbers of Iba1+ (activated microglia) were decreased in H-Apln and GFP groups compared with other injury groups. In sum, transplantation of iPSCs overexpressing apelin decreased microglial activation and regulated inflammatory cytokines in injured tissue, thus limiting tissue damage and pro-inflammatory reactions.

Although NSC activation is considered to play an important role in spontaneous self-repair, ${ }^{[64]} \mathrm{SCl}$ provides a hostile microenvironment for transplanted cells with regard to their survival. Thus, inducing endogenous NSC proliferation and differentiation is a promising strategy. We therefore examined whether administration of apelin could promote endogenous NSC proliferation using BrdU-incorporation experiments. BrdU+/Nestin+ cells represented activated endogenous NSCs after SCl. Our results show that transplantation of apelin overexpressed iPSCs promoted activation and proliferation of endogenous NSCs after $\mathrm{SCl}$, as well as repair of the injury.

We next identified newborn neurons and astrocytes by BrdU+/NeuN+ and BrdU+/GFAP+ immunofluorescence. Our results show that iPSCs overexpressing apelin promoted the survival of newborn neurons, but this phenomenon was not seen in other groups. In addition, we observed large number of newborn astrocytes in the injured groups, with the exception of the H-Apln group, similar to the results of in vitro experiments.

\section{Conclusion}

Altogether, our study demonstrates that apelin expression was decreased after $\mathrm{SCl}$ in both neurons and astrocytes, indicating a potential role in SCl process. To evaluate this hypothesis, we used iPSCs as a carrier to transplant apelin into the injured spinal cord tissue of rats, and observed the treatment effect. Our results show that administration of iPSCs overexpressing apelin repaired the disrupted architecture caused by SCl; improved motor function in the hindlimbs; reduced microglial and A1 astrocyte activation; regulated the expression of inflammatory cytokines; promoted the activation, proliferation, and differentiation of NSCs (both in vivo and in vitro); and, finally, resulted in functional recovery after SCI.

\section{Abbreviations}

ANOVA: Analysis of variance; NEC: Central nervous system; NSCs: endogenous neural stem cells; iPSCs: induced pluripotent stem cells; GFAP: Glial fibrillary acidic protein; IL: Interleukin; SCI: Spinal cord injury; PBS: Phosphate buffered saline; ELISA: Enzyme-linked immunosorbent assay; TNF-a囚Tumor necrosis factor alpha; HE: Hematoxylin-eosin; BBB: Basso, Beattie, and Bresnahan. 


\section{Declarations}

\section{Ethics approval and consent to participate}

Experimental protocols for animals in this study were approved by the Animal Care and Use Committee of Binzhou Medical University.

\section{Consent for publication}

No applicable.

\section{Availability of data and materials}

All data generated during and/or analysis during the current study are included in this published article.

\section{Competing interests}

The authors declare that they have no competing interests.

\section{Funding}

This work was supported by grants from the National Natural Science Foundation (Grant No. 81870985 and 81171142) and National Natural Science Foundation of Shandong Province (Grant No. ZR201702140355).

\section{Authors' contributions}

QL and FH conceived and designed the experiments. QL carried out most of the experiments and drafted the manuscript. CG, WX and QG participated in the animal model establishment. CZ and NZ analyzed the data. FH and LZ reviewed and rated the manuscript and participated in the final edition of the paper. All authors read and approved the final manuscript.

\section{Acknowledgements}

We would like to thank Zimeng Huang (Department of Medicine of Qingdao University, Qingdao University, Qingdao 266003, China) for her linguistic assistance.

\section{References}

1. WITIW CD F M. Acute Spinal Cord Injury [J]. Spinal disord tech, 2015, 28(6): 202-10.

2. ZHOU X, HE X, REN Y. Function of microglia and macrophages in secondary damage after spinal cord injury [J]. Neural Regen Res, 2014, 9(20): 1787-95.

3. JOHN C GENSEL D J D P G P. Spinal cord injury therapies in humans_ an overview of current clinical trials and their potential effects on intrinsic CNS macrophages [J]. Gensel, Donnelly \& Popovich, 
2011, 15(4): 505-18

4. REN Y, YOUNG W. Managing inflammation after spinal cord injury through manipulation of macrophage function [J]. Neural Plast, 2013, 2013(945034.

5. CODELUPPI S, SVENSSON C I, HEFFERAN M P, et al. The Rheb-mTOR pathway is upregulated in reactive astrocytes of the injured spinal cord [J]. J Neurosci, 2009, 29(4): 1093-104.

6. ZAMANIAN J L, XU L, FOO L C, et al. Genomic analysis of reactive astrogliosis [J]. J Neurosci, 2012, 32(18): 6391-410.

7. ANDERSON M A, BURDA J E, REN Y, et al. Astrocyte scar formation aids central nervous system axon regeneration [J]. Nature, 2016, 532(7598): 195-200.

8. JIANG D, GONG F, GE X, et al. Neuron-derived exosomes-transmitted miR-124-3p protect traumatically injured spinal cord by suppressing the activation of neurotoxic microglia and astrocytes [J]. J Nanobiotechnology, 2020, 18(1): 105.

9. TATEMOTO K, HOSOYA M, HABATA Y, et al. Isolation and characterization of a novel endogenous peptide ligand for the human apj receptor [J]. Biochemical and biophysical research communications, 1998, 251(471-6.

10. GU Q, ZHAI L, FENG X, et al. Apelin-36, a potent peptide, protects against ischemic brain injury by activating the PI3K/Akt pathway [J]. Neurochemistry International, 2013, 63(6): 535-40.

11. YANG Y, ZHANG X, CUI H, et al. Apelin-13 protects the brain against ischemia/reperfusion injury through activating PI3K/Akt and ERK1/2 signaling pathways [J]. Neuroscience Letters, 2014, 568(44-9.

12. ZENG X J, YU S P, ZHANG L, et al. Neuroprotective effect of the endogenous neural peptide apelin in cultured mouse cortical neurons [J]. Experimental Cell Research, 2010, 316(11): 1773-83.

13. XU Z, LI Z. Experimental Study on the Role of Apelin-13 in Alleviating Spinal Cord Ischemia Reperfusion Injury Through Suppressing Autophagy [J]. Drug Design, Development and Therapy, 2020, 14(1571-81.

14. J T, J D, R C, et al. New therapeutic targets for the development of positive inotropic agents [J]. Discov Med, 2011, 12(381e92.

15. JAPP A G, CRUDEN N L, BARNES G, et al. Acute cardiovascular effects of apelin in humans: potential role in patients with chronic heart failure [J]. Circulation, 2010, 121(16): 1818-27.

16. XU L, ZHOU S, FENG G Y, et al. Neural stem cells enhance nerve regeneration after sciatic nerve injury in rats [J]. Mol Neurobiol, 2012, 46(2): 265-74.

17. BERNDT M, LI Y, SEYEDHASSANTEHRANI N, et al. Fabrication and characterization of microspheres encapsulating astrocytes for neural regeneration [J]. ACS Biomater Sci Eng, 2017, 3(7): 1313-21.

18. TATOR A K A C H. Intrathecal administration of epidermal growth factor and fibroblast growth factor 2 promotes ependymal proliferation and functional recovery after spinal cord injury in adult rats [J]. JOURNAL OF NEUROTRAUMA, 2002, 19(223-38. 
19. PHILIP J. HORNER A E P, GERD KEMPERMANN, H. GEORG KUHN, THEO D. PALMER, JU“ RGEN WINKLER, LEON J.THAL, FRED H. GAGE. Proliferation and differentiation of progenitor cells throughout the_TISSUE ENGINEERINGintact adult rat spinal cord [J]. The Journal of Neuroscience, 2000, 20(6): 2218-28.

20. ZHANG L, WANG G, CHEN X, et al. Formyl peptide receptors promotes neural differentiation in mouse neural stem cells by ROS generation and regulation of PI3K-AKT signaling [J]. Sci Rep, 2017, 7(1): 206.

21. ASON B, CHEN Y, GUO Q, et al. Cardiovascular response to small-molecule APJ activation [J]. JCI Insight, 2020, 5(8):

22. HEATHER E. OLSON M D, GEMMA E. ROONEY, PH.D., LOUANN GROSS, JARRED J. NESBITT, B.S.,KATHERINE E. GALVIN, PH.D., ANDREW KNIGHT, PH.D., BINGKUN CHEN, M.D., PH.D.,MICHAEL J. YASZEMSKI, M.D., PH.D., AND ANTHONY J. WINDEBANK, M.D. Neural stem cell- and schwann cellloaded biodegradable polymer scaffolds support axonal regeneration in the transected spinal cord [J]. Tissue engineering, 2009, 15(1797-805.

23. XU F C Z L L, MEI X M. Culture of neural stem cells and the influencing factors [J]. Chinese journal of tissue engineering research, 2013,

24. SHENOY A, DANIAL M, BLELLOCH R H. Let-7 and miR-125 cooperate to prime progenitors for astrogliogenesis [J]. EMBO J, 2015, 34(9): 1180-94.

25. EK C J, HABGOOD M D, CALLAWAY J K, et al. Spatio-temporal progression of grey and white matter damage following contusion injury in rat spinal cord [J]. PLoS One, 2010, 5(8): e12021.

26. HASHIMOTO K, NAKASHIMA M, HAMANO A, et al. 2-carba cyclic phosphatidic acid suppresses inflammation via regulation of microglial polarisation in the stab-wounded mouse cerebral cortex [J]. Sci Rep, 2018, 8(1): 9715.

27. GARBUZOVA-DAVIS S, KURIEN C, THOMSON A, et al. Endothelial and Astrocytic Support by Human Bone Marrow Stem Cell Grafts into Symptomatic ALS Mice towards Blood-Spinal Cord Barrier Repair [J]. Sci Rep, 2017, 7(1): 884.

28. LIDDELOW S A, GUTTENPLAN K A, CLARKE L E, et al. Neurotoxic reactive astrocytes are induced by activated microglia [J]. Nature, 2017, 541(7638): 481-7.

29. DONALDSON D S, BRADFORD B M, ELSE K J, et al. Accelerated onset of CNS prion disease in mice co-infected with a gastrointestinal helminth pathogen during the preclinical phase [J]. Sci Rep, 2020, 10(1): 4554.

30. GAOJIAN T, DINGFEI Q, LINWEI L, et al. Parthenolide promotes the repair of spinal cord injury by modulating M1/M2 polarization via the NF-KB and STAT 1/3 signaling pathway [J]. Cell Death Discovery, 2020, 6(1):

31. WANG Y, CHENG X, HE Q, et al. Astrocytes from the contused spinal cord inhibit oligodendrocyte differentiation of adult oligodendrocyte precursor cells by increasing the expression of bone morphogenetic proteins [J]. J Neurosci, 2011, 31(16): 6053-8. 
32. STENUDD M, SABELSTROM H, FRISEN J. Role of endogenous neural stem cells in spinal cord injury and repair [J]. JAMA Neurol, 2015, 72(2): 235-7.

33. HE S, WANG Z, LI Y, et al. MicroRNA-92a-3p enhances functional recovery and suppresses apoptosis after spinal cord injury via targeting phosphatase and tensin homolog [J]. Biosci Rep, 2020, 40(5):

34. HE Y, LI M, WUJISIGULENG, et al. Zhenbao Pill reduces Treg cell proportion in acute spinal cord injury rats by regulating TUG1/miR-214/HSP27 axis [J]. Biosci Rep, 2018, 38(6):

35. TAYLOR X, CISTERNAS P, YOU Y, et al. A1 reactive astrocytes and a loss of TREM2 are associated with an early stage of pathology in a mouse model of cerebral amyloid angiopathy [J]. $\mathrm{J}$ Neuroinflammation, 2020, 17(1): 223.

36. LIDDELOW S A, BARRES B A. Reactive Astrocytes: Production, Function, and Therapeutic Potential [J]. Immunity, 2017, 46(6): 957-67.

37. XU W, LI T, GAO L, et al. Apelin-13/APJ system attenuates early brain injury via suppression of endoplasmic reticulum stress-associated TXNIP/NLRP3 inflammasome activation and oxidative stress in a AMPK-dependent manner after subarachnoid hemorrhage in rats [J]. Journal of Neuroinflammation, 2019, 16(1):

38. LIU Y, ZHANG T, WANG Y, et al. Apelin-13 attenuates early brain injury following subarachnoid hemorrhage via suppressing neuronal apoptosis through the GLP-1R/PI3K/Akt signaling [J]. Biochem Biophys Res Commun, 2019, 513(1): 105-11.

39. BAO H J, ZHANG L, HAN W C, et al. Apelin-13 attenuates traumatic brain injury-induced damage by suppressing autophagy [J]. Neurochem Res, 2015, 40(1): 89-97.

40. CHEN D, LEE J, GU X, et al. Intranasal delivery of Apelin-13 is neuroprotective and promotes angiogenesis after ischemic stroke in mice [J]. ASN Neuro, 2015, 7(5):

41. PAN Y, LI Q, YAN H, et al. Apela improves cardiac and renal function in mice with acute myocardial infarction [J]. J Cell Mol Med, 2020, 24(18): 10382-90.

42. AMINYAVARI S, ZAHMATKESH M, KHODAGHOLI F, et al. Anxiolytic impact of Apelin-13 in a rat model of Alzheimer's disease: Involvement of glucocorticoid receptor and FKBP5 [J]. Peptides, 2019, 118(170102.

43. LUO H, XIANG Y, QU X, et al. Apelin-13 Suppresses Neuroinflammation Against Cognitive Deficit in a Streptozotocin-Induced Rat Model of Alzheimer's Disease Through Activation of BDNF-TrkB Signaling Pathway [J]. Frontiers in Pharmacology, 2019, 10 (

44. ZHU J, DOU S, JIANG Y, et al. Apelin-13 protects dopaminergic neurons in MPTP-induced Parkinson's disease model mice through inhibiting endoplasmic reticulum stress and promoting autophagy [J]. Brain Research, 2019, 1715(203-12.

45. ZHU J, GAO W, SHAN X, et al. Apelin-36 mediates neuroprotective effects by regulating oxidative stress, autophagy and apoptosis in MPTP-induced Parkinson's disease model mice [J]. Brain Research, 2020, 1726(

46. HU W, JIANG W, YE L, et al. Prospective evaluation of the diagnostic value of plasma apelin 12 levels for differentiating patients with moyamoya and intracranial atherosclerotic diseases [J]. Sci Rep, 
2017, 7(1): 5452.

47. MELGAR-LESMES P, PERRAMON M, JIMENEZ W. Roles of the Hepatic Endocannabinoid and Apelin Systems in the Pathogenesis of Liver Fibrosis [J]. Cells, 2019, 8(11):

48. VAFAEI-NEZHAD S, NIKNAZAR S, NOROUZIAN M, et al. Therapeutics effects of [Pyr1] apelin-13 on rat contusion model of spinal cord injury: An experimental study [J]. J Chem Neuroanat, 2021, 113(101924.

49. XIN Q, CHENG B, PAN Y, et al. Neuroprotective effects of apelin-13 on experimental ischemic stroke through suppression of inflammation [J]. Peptides, 2015, 63(55-62.

50. SERPOOSHAN V, SIVANESAN S, HUANG X, et al. [Pyr1]-Apelin-13 delivery via nano-liposomal encapsulation attenuates pressure overload-induced cardiac dysfunction [J]. Biomaterials, 2015, 37(289-98.

51. SHIGA Y, SHIGA A, MESCI P, et al. Tissue-type plasminogen activator-primed human iPSC-derived neural progenitor cells promote motor recovery after severe spinal cord injury [J]. Sci Rep, 2019, 9(1): 19291.

52. YAO M, YANG L, WANG J, et al. Neurological recovery and antioxidant effects of curcumin for spinal cord injury in the rat: a network meta-analysis and systematic review [J]. J Neurotrauma, 2015, 32(6): 381-91.

53. LIU F T, XU S M, XIANG Z H, et al. Molecular hydrogen suppresses reactive astrogliosis related to oxidative injury during spinal cord injury in rats [J]. CNS Neurosci Ther, 2014, 20(8): 778-86.

54. ALMUTIRI S, BERRY M, LOGAN A, et al. Non-viral-mediated suppression of AMIGO3 promotes disinhibited NT3-mediated regeneration of spinal cord dorsal column axons [J]. Sci Rep, 2018, 8(1): 10707.

55. KOSTYK S K, POPOVICH P G, STOKES B T, et al. Robust axonal growth and a blunted macrophage response are associated with impaired functional recovery after spinal cord injury in the MRL/MpJ mouse [J]. Neuroscience, 2008, 156(3): 498-514.

56. ALDER J, FUJIOKA W, GIARRATANA A, et al. Genetic and pharmacological intervention of the p75NTR pathway alters morphological and behavioural recovery following traumatic brain injury in mice [J]. Brain Inj, 2016, 30(1): 48-65.

57. RIEW T R, KIM S, JIN X, et al. Osteopontin and its spatiotemporal relationship with glial cells in the striatum of rats treated with mitochondrial toxin 3-nitropropionic acid: possible involvement in phagocytosis [J]. J Neuroinflammation, 2019, 16(1): 99.

58. CLARKE L E, LIDDELOW S A, CHAKRABORTY C, et al. Normal aging induces A1-like astrocyte reactivity [J]. Proc Natl Acad Sci U S A, 2018, 115(8): E1896-E905.

59. ZARB Y, WEBER-STADLBAUER U, KIRSCHENBAUM D, et al. Ossified blood vessels in primary familial brain calcification elicit a neurotoxic astrocyte response [J]. Brain, 2019, 142(4): 885-902.

60. CHEN W K, FENG L J, LIU Q D, et al. Inhibition of leucine-rich repeats and calponin homology domain containing 1 accelerates microglia-mediated neuroinflammation in a rat traumatic spinal cord injury model [J]. J Neuroinflammation, 2020, 17(1): 202. 
61. MICHELS M, AVILA P, PESCADOR B, et al. Microglial Cells Depletion Increases Inflammation and Modifies Microglial Phenotypes in an Animal Model of Severe Sepsis [J]. Mol Neurobiol, 2019, 56(11): 7296-304.

62. GORDON S, MARTINEZ F O. Alternative activation of macrophages: mechanism and functions [J]. Immunity, 2010, 32(5): 593-604.

63. RAMACHANDRAN P, PELLICORO A, VERNON M A, et al. Differential Ly-6C expression identifies the recruited macrophage phenotype, which orchestrates the regression of murine liver fibrosis [J]. Proc Natl Acad Sci U S A, 2012, 109(46): E3186-95.

64. KIM C, KIM H J, LEE H, et al. Mesenchymal Stem Cell Transplantation Promotes Functional Recovery through MMP2/STAT3 Related Astrogliosis after Spinal Cord Injury [J]. Int J Stem Cells, 2019, 12(2): $331-9$.

\section{Figures}
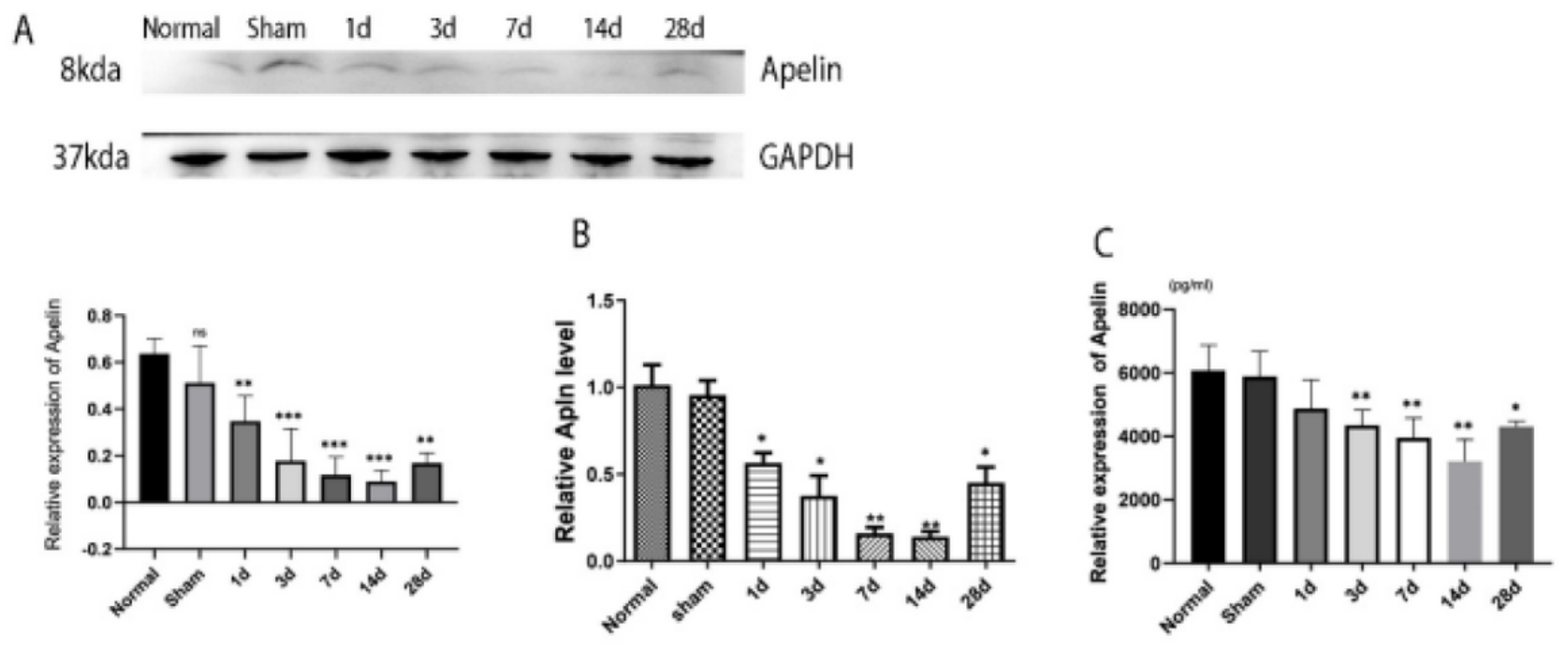

\section{Figure 1}

Expression of apelin decreased after spinal cord injury (SCI). (A) Spinal cord sections obtained from healthy uninjured rats (control) and $\mathrm{SCl}$ rats on days 1, 3, 7, 14 and 28 after $\mathrm{SCl}$; apelin protein levels were normalized to that of GAPDH after western blotting. (B) Gene expression of apelin was quantified in total RNA isolated from spinal cord tissue using qRT-PCR with specific primers; GAPDH was used as a loading control for qRT-PCR. (C) Serum apelin was assayed by ELISA after $S C l .\left({ }^{*} p<0.05,{ }^{\star *} p<0.01\right.$ vs. control group, mean $\pm \mathrm{SD})$. 

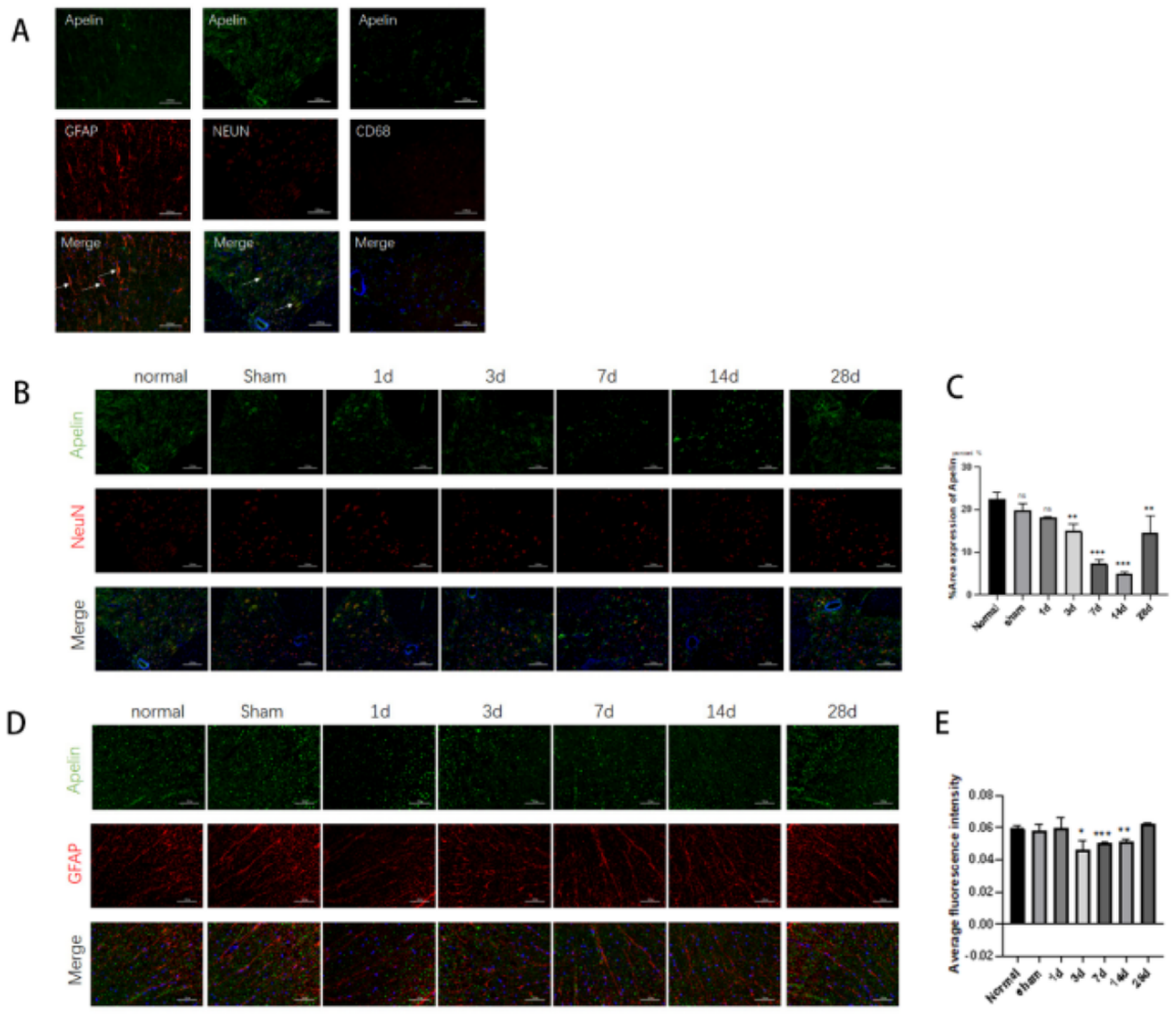

Figure 2

Changes of apelin expression in spinal cord tissues during spinal cord injury. (A) Immunofluorescence imaging showing co-localization of apelin (green) with markers of specific cells types including NeuN (red, neurons), GFAP (red, astrocytes), and CD68 (red, microglia) after 1 day post injury (dpi); nuclei were counterstained with DAPI (blue). Co-localization appears yellow in the merged image. Bar $=250 \mu \mathrm{m}$. $(\mathrm{B}$ and D) Immunofluorescence staining showing apelin expression in spinal cord tissue in neurons (red) and astrocytes (red) at different time points. Bar $=250 \mu \mathrm{m}$. (C and $\mathrm{E})$ The immunofluorescence quantitative analysis of apelin ( ${ }^{*}<0.05,{ }^{\star \star} p<0.01$ vs. control group, mean $\left.\pm S D\right)$. 
A
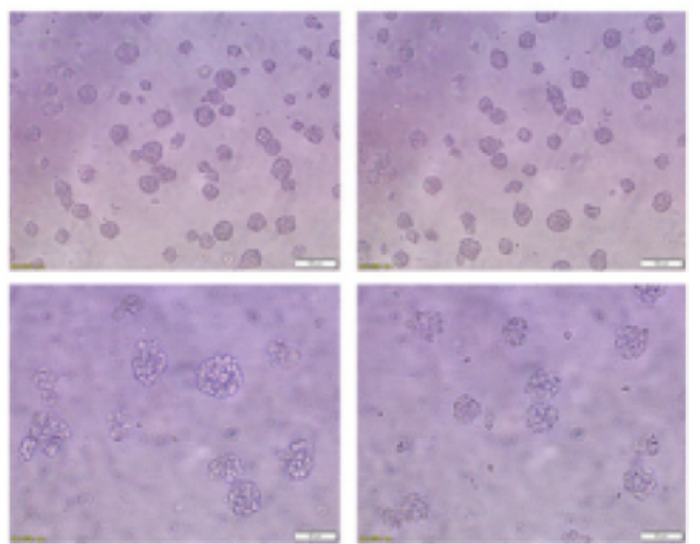

B
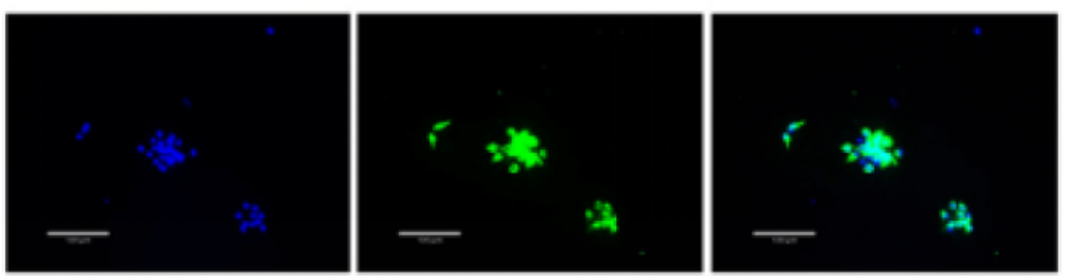

c
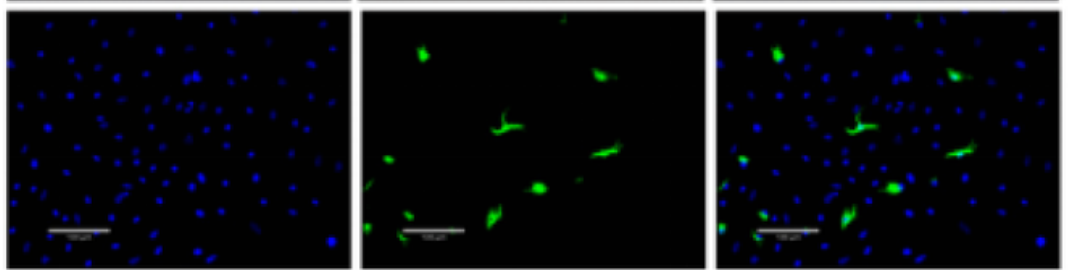

D
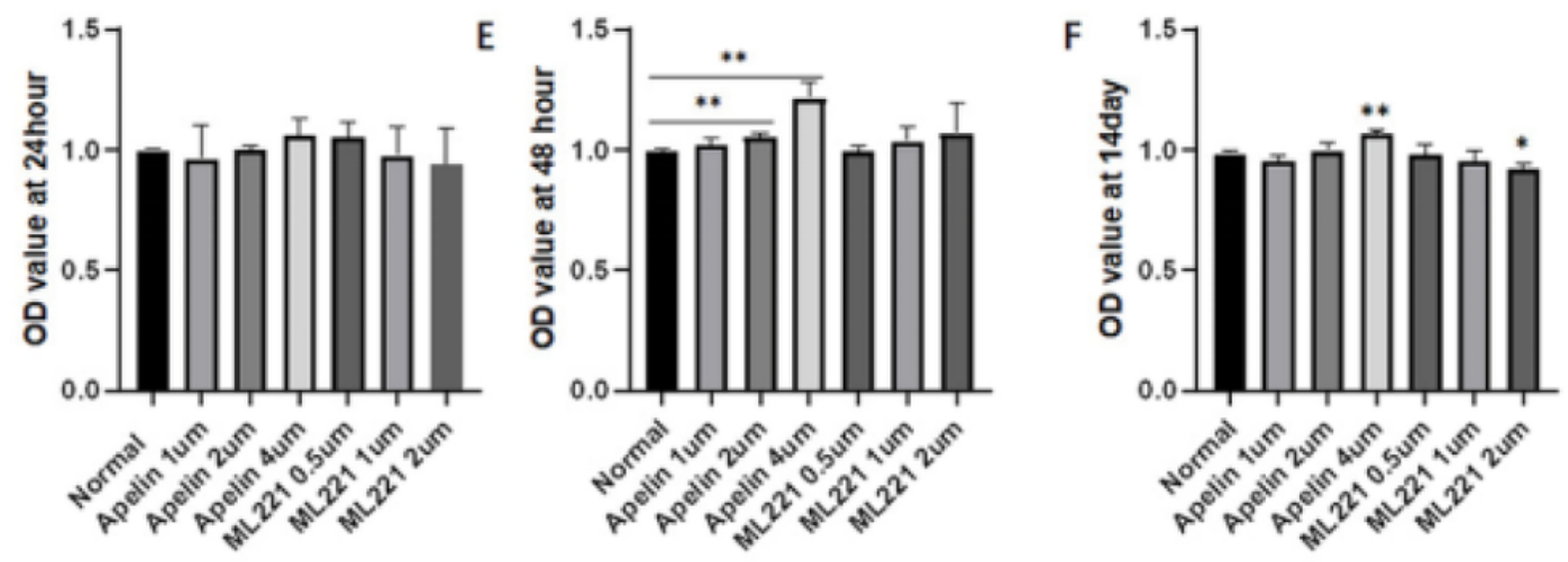

\section{Figure 3}

Identification of primary cultured neural stem cells (NSCs) and evaluation of their proliferation after administration of apelin or its inhibitor ML221. (A) Morphology of NSCs under a light microscope, bar = $50 \mu \mathrm{m}$. (B) Identification of primary cultured NSCs, bar $=130 \mu \mathrm{m}$. (C) Multi-differentiation potential of NSCs was assessed by GFAP+ cells (green) after $14 \mathrm{~d}$, bar $=130 \mu \mathrm{m}$. Proliferation of NSCs under different dosages of apelin and its inhibitor ML221 were determined by CCK8 assay at $24 \mathrm{~h}$ (D), $48 \mathrm{~h}$ (E), and $14 \mathrm{~d}(\mathrm{~F})$ after $\mathrm{SCl}\left({ }^{\star} \mathrm{p}<0.05,{ }^{\star \star} \mathrm{p}<0.01\right.$ vs. control group, mean $\left.\pm \mathrm{SD}\right)$. 

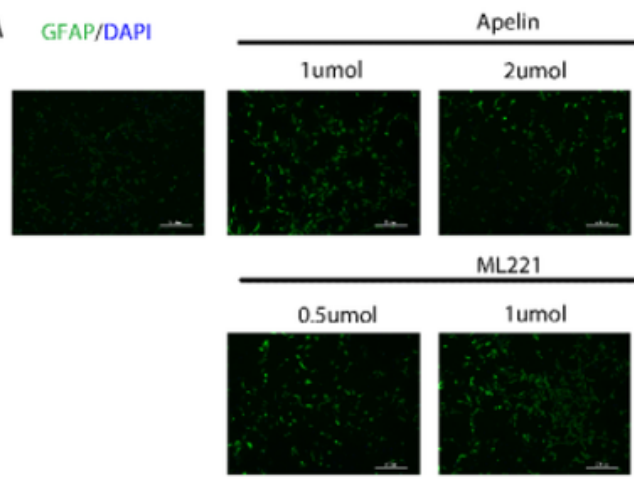

ML221

1umol

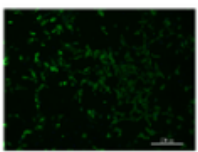

Apelin
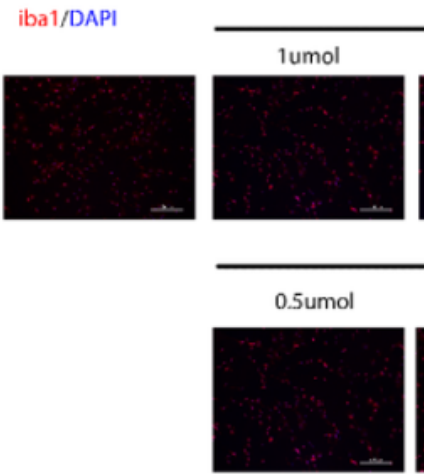

ML221

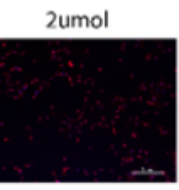

1 umol

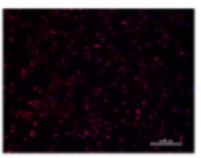

2umol

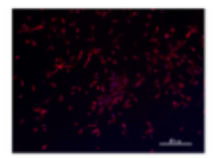

C Olig2/DAPI
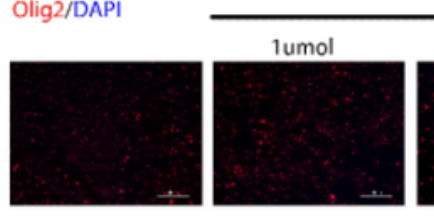

Apelin

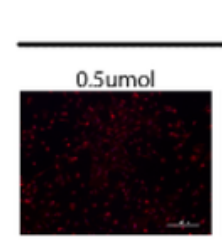

D
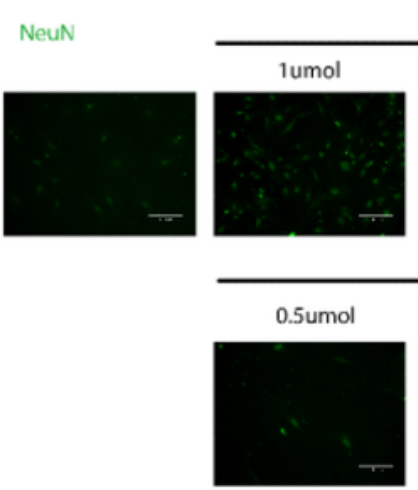

Apelin
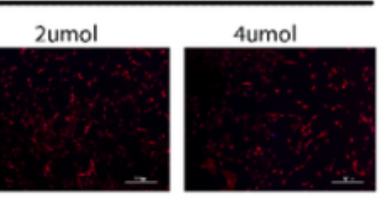

ML221

1umol

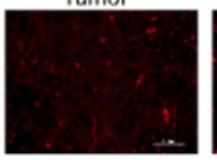

2umol

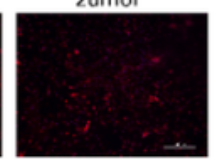

2umol

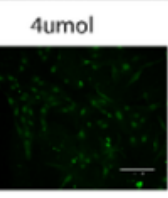

ML221

1umol

2umol
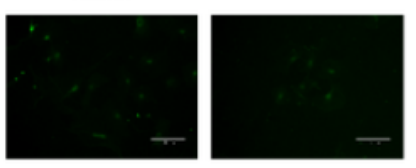
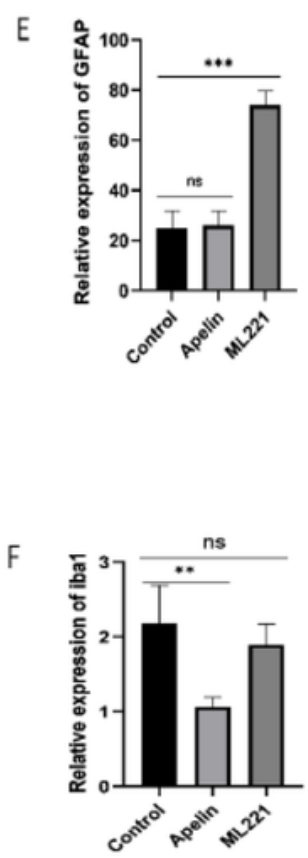

G
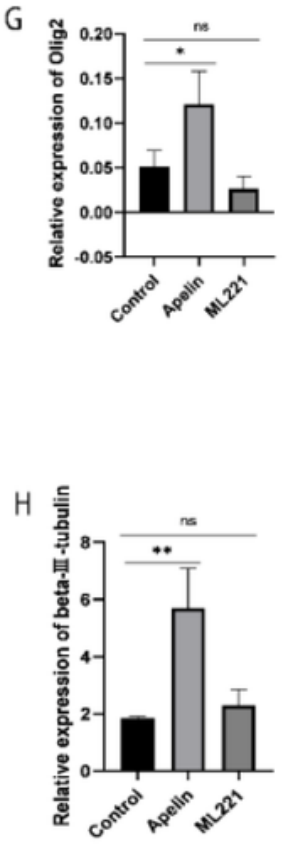

\section{Figure 4}

Apelin and ML221 promoted differentiation of NSCs. Differentiated cell types were identified by expression of specific markers via immunofluorescence: (A) GFAP (green) for astrocytes, (B) lba1 (red) for microglia, (C) Olig2 (red) for oligodendroglia, and (D) NeuN (green) for neurons; nuclei were counterstained with DAPI (blue). (E-H) RT-PCR results indicating different types of cells differentiated from NSCs following induction by apelin and ML221. Primers for four targets were used: GFAP (astrocytes), Iba1 
(microglia), Olig2 (oligodendroglia), and beta-three-tubulin (neurons); a specific primer for GAPDH was also included in reactions $\left({ }^{\star} p<0.05,{ }^{\star \star} p<0.01,{ }^{\star \star *} p<0.001\right.$ vs. control group, mean $\pm S D$ ).

\section{Figure 5}

iPSC transplantation promoted morphological and functional recovery after SCl. A. HE staining of spinal cord tissue at $14 \mathrm{~d}$ after injury, bar $=200 \mu \mathrm{m}$ or $110 \mu \mathrm{m}$. B, LFB staining was performed to identify demyelination at $14 \mathrm{~d}$, bar $=200 \mu \mathrm{m}$ or $110 \mu \mathrm{m}$. C and D. Nissl staining to assess the loss of neurons at $14 \mathrm{~d}$, and statistical graph showing numbers of Nissl bodies in each group ( ${ }^{\star \star} \mathrm{p}<0.01,{ }^{\star \star \star} p<0.001 \mathrm{vs}$. Sham control group, mean \pm SD). E. Neurological function assessed by BBB score from 0 to $14 \mathrm{~d}$ after

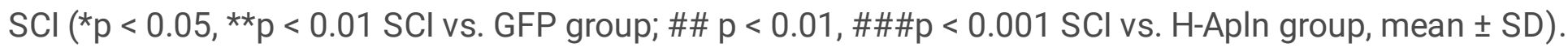
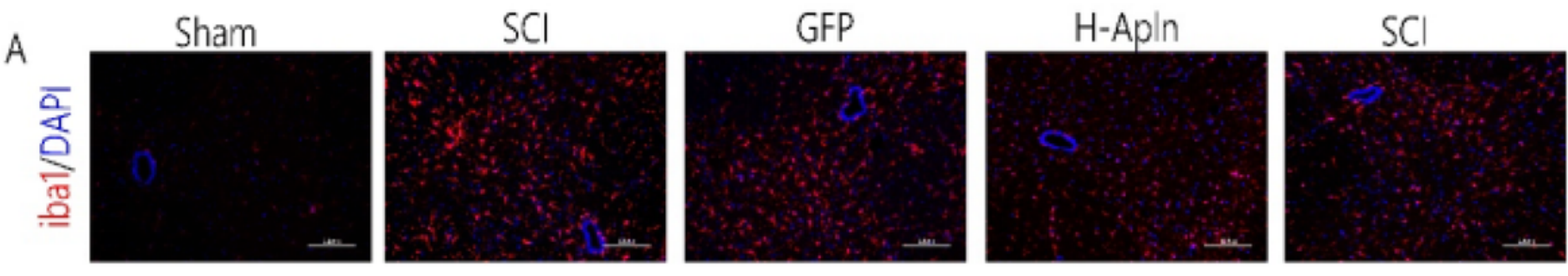

B
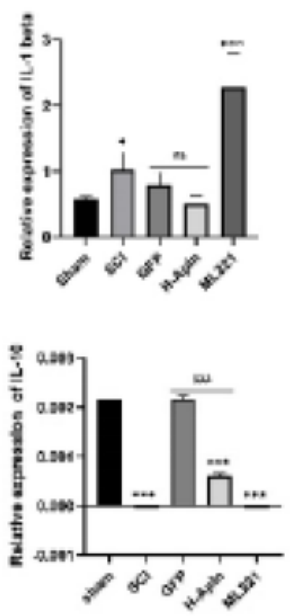
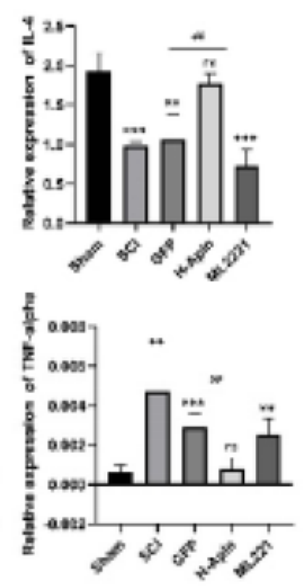
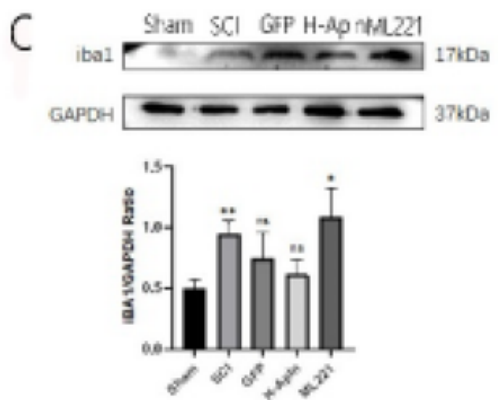

\section{Figure 6}

Cell administration alleviated microglial activation after SCI. A. Quantification of microglia (Iba1, red) determined by immunofluorescence staining, scale $=120 \mu \mathrm{m}$. B. Relative expression of pro-inflammatory (IL-1 $\beta$ and TNF- $\alpha$ ) and anti-inflammatory (IL-4 and IL-10) cytokines secreted by microglia detected by qRTPCR at $14 \mathrm{~d}$ post injury ( ${ }^{*} p<0.05,{ }^{\star \star} p<0.01,{ }^{\star \star *} p<0.001$ vs. Sham group; \#\#p $<0.01$, \#\#\#p $<0.001$ GFP vs. H-Apln, mean \pm SD). C. Protein expression of Iba1 (microglial marker) was determined by western blotting. ( ${ }^{*} p<0.05$, ${ }^{\star *} p<0.01$ vs. Sham group, mean $\pm S D$ ). D, Relative mRNA expression of lba1 was measured by $q R T-P C R(* \star p<0.01, * \star * p<0.001$ vs. Sham group, mean $\pm S D)$. 

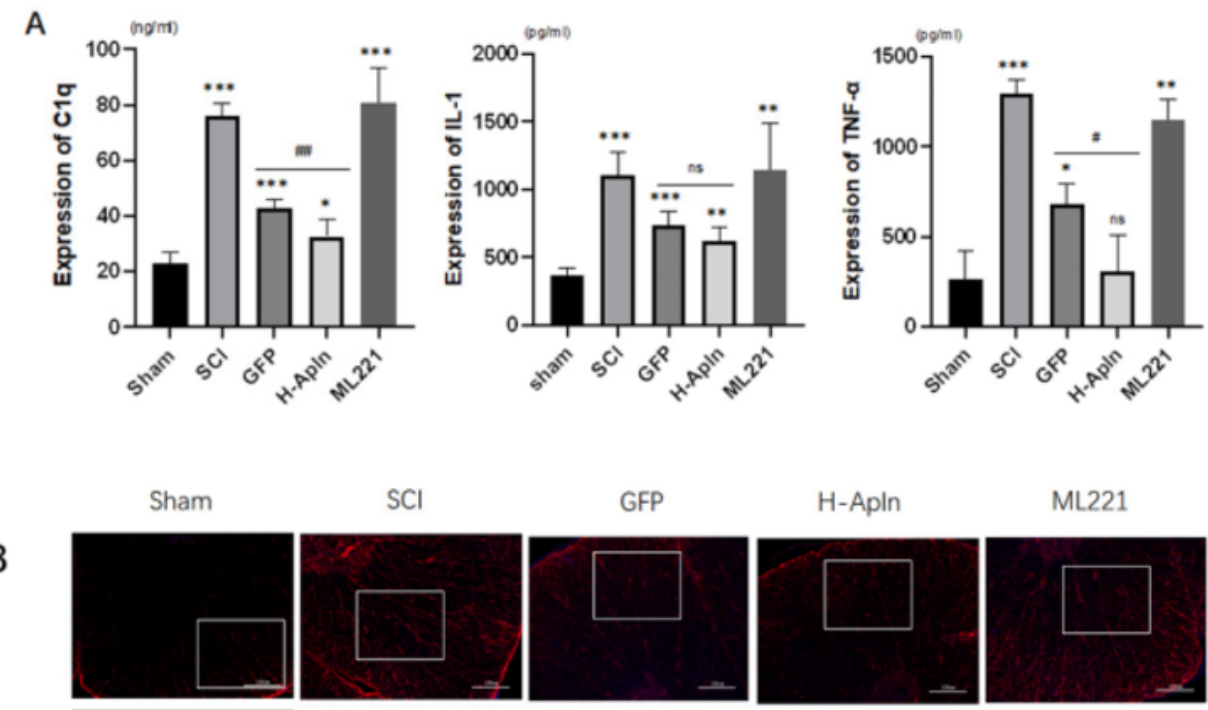

GFP

H-Apln

ML221
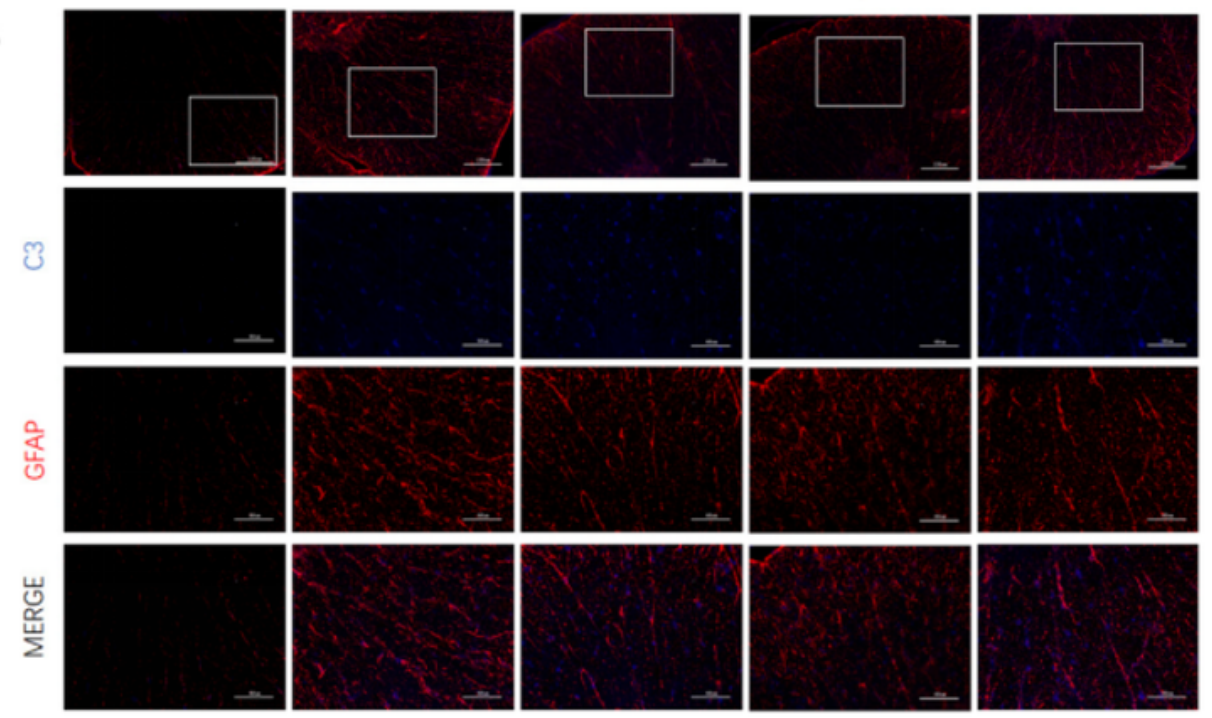

C
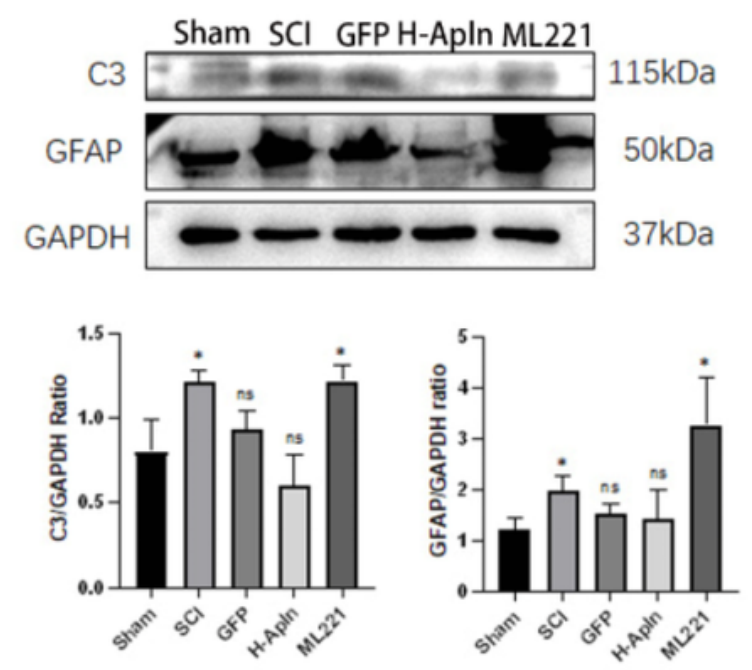

\section{Figure 7}

Cell transplantation reduced activation of A1 astrocytes. A. Protein expression levels of pro-inflammation cytokines that promote A1 astrocyte activation were examined by RT-PCR post $\mathrm{SCl}$. $\left({ }^{\star} \mathrm{p}<0.05,{ }^{*} \mathrm{p}<0.01\right.$, ${ }^{* \star *} \mathrm{p}<0.001$ vs. Sham group, mean \pm SD). B. A1 astrocytes in spinal cord tissue double-labeled by GFAP (red) and C3 (blue); the sham group was used as a control, scale $=120 \mu \mathrm{m}$ or $60 \mu \mathrm{m}$. C. Western blotting of relative expression of GFAP and C3 in each group ( ${ }^{\star} p<0.05$ vs. Sham group, mean \pm SD). 


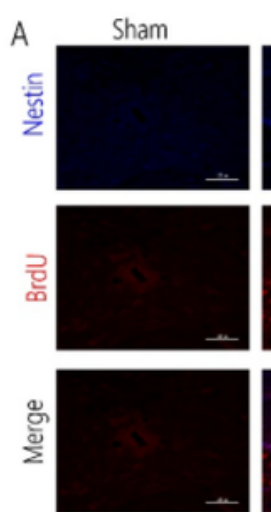

B
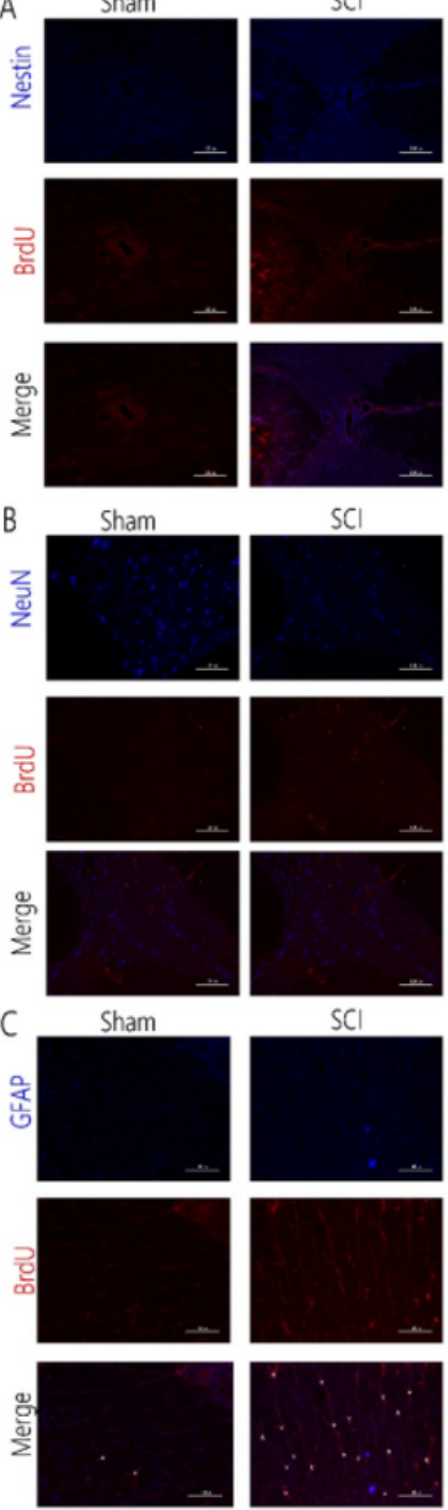

SCI
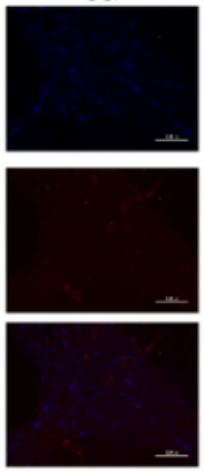

SCI
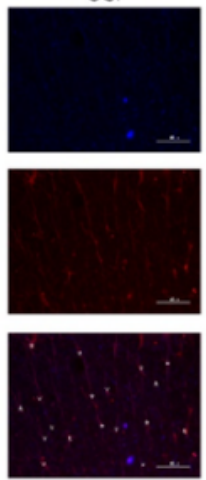

D
GFP
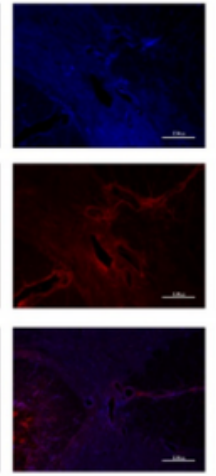

GFP

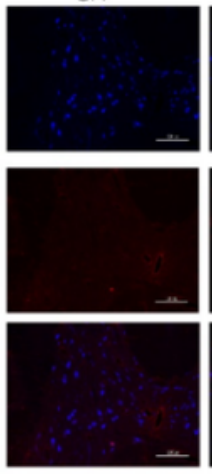

GFP
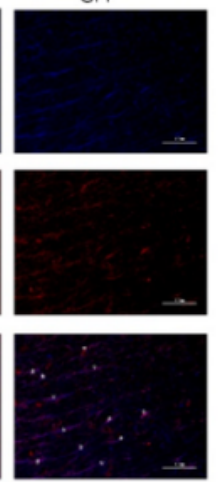

H-Apln
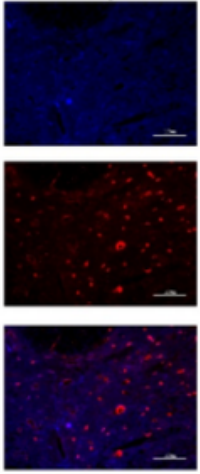

H-Apln

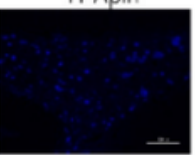

ML221
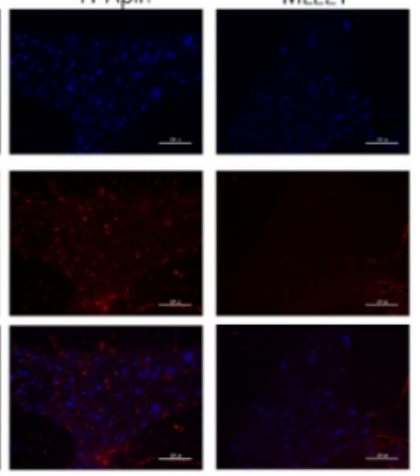

H-Apln
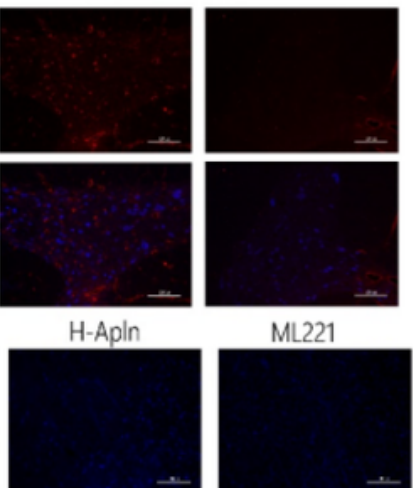

ML221
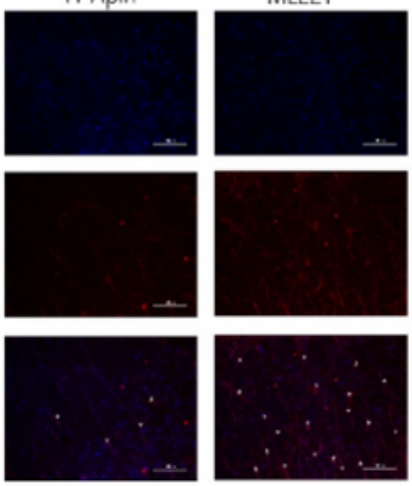

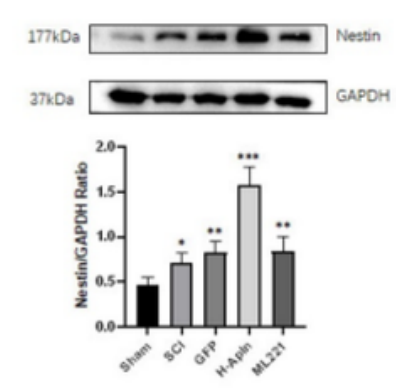

Figure 8

Activation and differentiation of endogenous neural stem cells after transplantation of iPSCs. A. Activation and proliferation of endogenous NSCs was detected using BrdU/nestin coimmunofluorescence at 14 days after $\mathrm{SCl}$ in each group. $\mathrm{B}$ and $\mathrm{C}$. Double-immunofluorescence staining of NeuN (red) and BrdU (blue, cell proliferation marker) to identify the differentiation of newly formed cells towards neurons (arrowheads), or GFAP (blue) and BrdU (red) to identify newly formed astrocytes 
(asterisk). D. Western blotting analysis of nestin protein expression $\left({ }^{\star} p<0.05,{ }^{*} p<0.01,{ }^{\star \star *} p<0.001\right.$ vs. Sham group, mean $\pm \mathrm{SD}$ ). 